\title{
Toddler diarrhoea and prostaglandins
}

\author{
J A DODGE, I A HAMDI, G M BURNS, AND Y YAMASHIRO \\ Department of Child Health, Welsh National School of Medicine
}

SUMMARY Chronic, non-specific 'toddler diarrhoea' in young children is often accompanied by an increase in plasma prostaglandins, particularly $\mathrm{PGF}_{2} \alpha$. Although treatment with drugs is generally unnecessary, aspirin is effective in most children with high prostaglandins, and treatment with loperamide is effective in most children with toddler diarrhoea, regardless of the plasma prostaglandin levels. The source of the increased plasma prostaglandins is not known.

Persistent diarrhoea-without failure to thrive, abnormal physical signs, demonstrable pathogens, or evidence of malabsorption-is a common symptom in the young child. It is variously described as chronic, non-specific diarrhoea, irritable bowel syndrome, or toddler diarrhoea. The benign nature of the problem is generally evident from clinical examination, and further investigation is not usually indicated if the child is growing normally. ${ }^{1}$

We previously reported a child who had a severe form of non-specific diarrhoea and was found to have high circulating prostaglandin levels, who responded well to treatment with prostaglandin synthetase inhibitors (aspirin and indomethacin). Administration and withdrawal of the drugs produced remissions and relapses. ${ }^{2}$ We have now investigated a further 30 children with the syndrome, and compared their plasma prostaglandin levels with those of 26 healthy controls. We have also evaluated the effect of prostaglandin synthetase inhibitors.

\section{Investigations}

Thirty-one patients were studied in whom the diagnosis of 'toddler diarrhoea' was made by a process of exclusions. All were in excellent general health, but had a history of watery stools for at least 3 months. The stools were examined for pathogens and parasites, and results were negative in each case. The growth pattern in all cases was normal. The stools were watery, not greasy, and did not contain reducing substances. Jejunal biopsy was carried out in 8 instances, and both the histological appearances and disaccharidase levels were within the normal range. The remainder of the patients were not biopsied, because we could not justify invasive tests in children who were thriving normally and suffering from a condition which is usually treated with nothing more than reassurance.

Plasma prostaglandin levels were measured in all instances: PGF $\alpha$ in all 31, and $\mathrm{PGE}_{2}$ in 22. A group of 26 children without gastrointestinal symptoms, admitted to hospital for operations such as herniorrhaphy, or undergoing investigations for short stature or enuresis, in whom no organic disease was discovered and from whom blood was being taken for other routine purposes, was used as a control. Venous samples were taken into heparinised tubes, and the plasma separated by centrifugation at once. Extraction of prostaglandins from the plasma was carried out after acidification with a formic acid/ ethanol mixture by 3 extractions with petroleum ether, and 2 with chloroform. The extract was dried under nitrogen, dissolved in phosphate buffer at $\mathrm{pH} 7 \cdot 4$, and stored in ethanol at $-20^{\circ} \mathrm{C}$ for up to 2 weeks before radioimmunoassay for $\mathrm{PGE}_{2}$ and PGF $\alpha$. Antisera used for the assays were obtained from the Institut Pasteur, Paris. Radioimmunoassays for the two prostaglandins were performed in triplicate on each sample, and repeated in most cases. $^{3}$ Good agreement was obtained between the individual measurements on samples from each patient and the figures presented represent mean values. A trial of therapy with aspirin, and with loperamide, was also carried out on some of these patients.

\section{Results}

The mean circulating PGF $\alpha$ in the normal children was $139.0 \mathrm{pg} / \mathrm{ml}$. In the patients with toddler diarrhoea, the mean was $452.9 \mathrm{pg} / \mathrm{ml}$. All 26 of the controls had levels below $300 \mathrm{pg} / \mathrm{ml}$, but 17 of the 31 patients exceeded this upper limit of normal (Fig. 1). 
The difference between the two groups was statistically significant $(\mathrm{P}<0 \cdot 01)$.

In the case of $\mathrm{PGE}_{2}$, although mean levels in the patients were slightly higher than in the controls (79.7 compared with $48.7 \mathrm{pg} / \mathrm{ml}$ ) most came within the normal range and no statistically significant differences were observed (Fig. 2).

The effects of aspirin (600-1200 mg daily), a

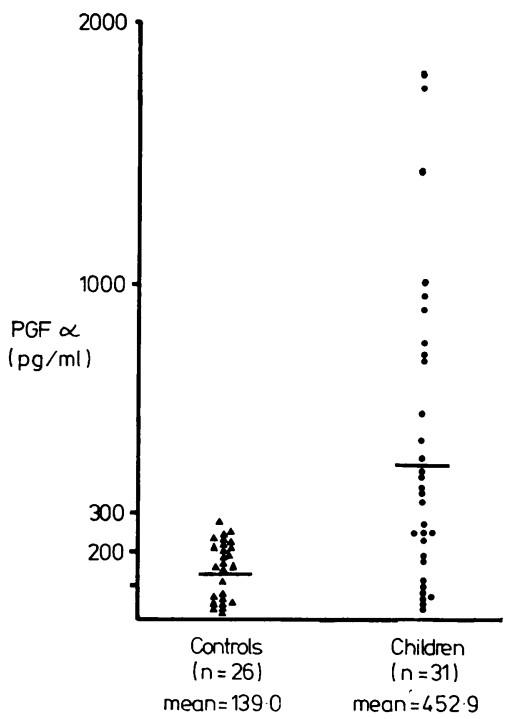

Fig. 1 Levels of PGF $\alpha$ in the patients compared with those in the controls.

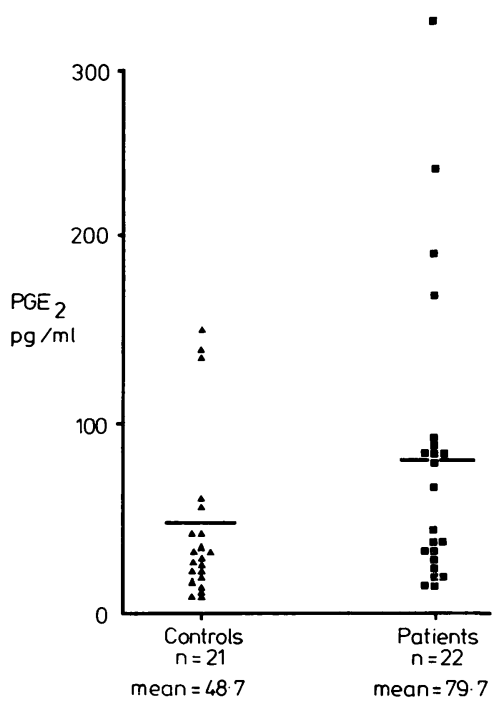

Fig. 2 Levels of $P G E_{2}$ in the patients compared with those in the controls. prostaglandin synthetase inhibitor, were observed in 14 patients, including 6 who had raised PGF $\alpha$ levels. A good clinical response, as judged by frequency and consistency of stools, was obtained in 5 of these, 4 of whom also responded to loperamide. Three out of 8 patients with normal prostaglandin levels did not respond to aspirin, but in all of these loperamide $(0 \cdot 1 \mathrm{mg} / \mathrm{kg}$ a day) was effective. Twelve patients were treated with loperamide in a double-blind cross-over trial, using ascorbic acid as a placebo. There was a pronounced improvement in both frequency and consistency of the stools in 10 cases, including 5 of the 6 with increased prostaglandins. ${ }^{4}$

We have since extended our experience with this drug and found it to be effective in those with normal prostaglandin levels as well as in those with hyperprostaglandinaemia. Measurement of plasma prostaglandin levels before and after treatment with aspirin showed a reduction after treatment in every case, but loperamide had little effect on plasma prostaglandin levels.

\section{Discussion}

Diarrhoea is an important side effect of exogenous $\mathrm{PGF}_{2} \alpha$ given to initiate labour or abortion. Excessive production of prostaglandins has also been implicated as the cause of the diarrhoea associated with medullary carcinoma of the thyroid, neural crest tumours, and, more controversially, cholera. Recently, high concentrations of prostaglandins have been demonstrated in the upper intestinal fluid of some adults with chronic, non-specific diarrhoea (irritable bowel syndrome). The patients responded to treatment with the prostaglandin synthetase inhibitor indomethacin. ${ }^{5}$ We have found that aspirin, which is also an inhibitor of prostaglandin synthesis, is immediately and strikingly effective in the treatment of toddler diarrhoea in nearly all patients who have increased plasma prostaglandins. Although it was not given systematically to all the others, we have noted that it is not always effective in patients with toddler diarrhoea, although those who respond are otherwise clinically indistinguishable from non-responders. In nearly all our child patients, loperamide was an effective antidiarrhoeal agent. ${ }^{4}$ This drug, whose exact mode of action is uncertain, is taken up by opiate receptors and has been shown to prevent diarrhoea induced by administration of prostaglandins in both animals and man. ${ }^{6}$ It has also been shown to reverse the secretory state induced by prostaglandins or cholera toxin administration to rats with everted loops of jejunum. ${ }^{78}$

The source of the increased plasma prostaglandins in these children is not known. It is possible 
that the prostaglandins are locally produced in the gastrointestinal tract in excess, and that sufficient are absorbed to raise circulating levels above normal. We have no evidence that this is the case, but if it is so, one would expect to find increased plasma prostaglandin levels in conditions such as ulcerative colitis, where there is known to be local gut inflammation and where significant amounts of prostaglandins are found in the stools. ${ }^{9}$

A second possibility is that excessive prostaglandins are produced elsewhere in the body, and the increased amounts found in the circulation stimulate the gastrointestinal tract. There is no evidence for this in these children. In conditionssuch as medullary carcinoma of the thyroid-in which large amounts of prostaglandins are produced in the gland, chronic diarrhoea may be an important symptom; $;^{10}$ but no association between the irritable bowel syndrome and a source of prostaglandins outside the gut has been reported.

Finally, normal amounts of prostaglandins may be produced in the gut and elsewhere, absorbed in normal amounts into the circulation, but inadequately removed in the lungs and liver. The spontaneous improvement which is seen in children with toddler diarrhoea would be in keeping with the suggestion that maturation of a metabolic pathway may occur. The fact that both aspirin and loperamide are effective antidiarrhoeal agents in some patients whose plasma prostaglandins are normal suggests that prostaglandins are important mediators of this type of diarrhoea and that they are produced at a local level in the gut, possibly in response to immunological stimulation.

Further studies of prostaglandin metabolism in adults with functional diarrhoea are indicated, and are not subject to the same practical and ethical constraints which apply to investigations in children.

G M B was supported by a grant from the Cystic Fibrosis Research Trust.

\section{References}

1 Anonymous. How necessary are elimination diets in childhood? Br Med J 1980; 280: 138.

2 Dodge J A, Hamdi I, Walker S. Prostaglandin-induced diarrhoea. Arch Dis Child 1977; 52: 800-2.

3 Hamdi I. Prostaglandins in some childhood diseases. PhD thesis, University of Wales 1979.

4 Dodge J A. Chronic non-specific diarrhoea in children. In: Gough D. The control of diarrhoea in clinical practice. Proceedings of an International Symposium held by the Janssen Research Foundation at the Royal Society of Medicine, London, July 1978. Royal Society of Medicine International Congress and Symposium Series No 5. London: Royal Society of Medicine/Academic Press, 1978: 31-4.

5 Rask-Madsen J, Bukhave K. Indomethacin-responsive diarrhoea in irritable-bowel syndrome (abstract). Gut 1978; 19A : 448-9.

6 Karim S M, Adaikan P G. The effect of loperamide on prostaglandin-induced diarrhoea in rat and man. Prostaglandins 1977; 13: 321-32.

7 Sandhu B, Tripp J H, Harries J T. A new look at loperamide (abstract). Arch Dis Child 1980; 55: 161.

8 Sandhu B, Tripp J H, Candy D C A, Harries J T. Letter: Loperamide inhibits cholera-toxin-induced smallintestinal secretion. Lancet 1979; ii: 689-90.

${ }^{9}$ Gould S R. Letter: Prostaglandins, ulcerative colitis, and sulphasalazine. Lancet 1975 ; ii: 988.

10 Williams E D, Karim S M M, Sandler M. Prostaglandin secretion by medullary carcinoma of the thyroid. Lancet 1968 ; i: $22-3$.

Correspondence to Dr J A Dodge, Department of Child Health, Welsh National School of Medicine, Heath Park, Cardiff CF4 4XN.

Received 11 June 1980 